\title{
Deletion at an 1q24 locus reveals a critical role of long noncoding RNA DNM3OS in skeletal development
}

\author{
Ting-ting Yu ${ }^{1}{ }^{\infty}$, Qiu-fan $\mathrm{Xu}^{1 \dagger}$, Si-Yang Li ${ }^{1 \dagger}$, Hui-jie Huang ${ }^{1}$, Sarah Dugan², Lei Shao', Jennifer A. Roggenbuck ${ }^{3}$, \\ Xiao-tong Liu', Huai-ze Liu', Betsy A. Hirsch ${ }^{4}$, Shen Yue ${ }^{1 *}$, Chen Liu ${ }^{1 *}$ and Steven Y. Cheng ${ }^{1 *}$
}

\begin{abstract}
Background: Skeletal development and maintenance are complex processes known to be coordinated by multiple genetic and epigenetic signaling pathways. However, the role of long non-coding RNAs (IncRNAs), a class of crucial epigenetic regulatory molecules, has been under explored in skeletal biology.

Results: Here we report a young patient with short stature, hypothalamic dysfunction and mild macrocephaly, who carries a maternally inherited $690 \mathrm{~kb}$ deletion at Chr.1q24.2 encompassing a noncoding RNA gene, DNM3OS, embedded on the opposite strand in an intron of the DYNAMIN 3 (DNM3) gene. We show that InCRNA DNM3OS sustains the proliferation of chondrocytes independent of two co-cistronic microRNAs miR-199a and miR-214. We further show that nerve growth factor (NGF), a known factor of chondrocyte growth, is a key target of DNM3OS-mediated control of chondrocyte proliferation.
\end{abstract}

Conclusions: This work demonstrates that DNM3OS is essential for preventing premature differentiation of chondrocytes required for bone growth through endochondral ossification.

Keywords: Skeletal abnormalities, 1q24, IncRNA, DNM3OS, Nerve growth factor

\section{Background}

Development of the skeletal system that supports body structures and maintenance of its homeostatic state are highly complex processes orchestrated by an elaborate array of gene activities. A frequently occurring developmental skeletal abnormally is short stature, which can arise as part of systemic diseases [1-3]. Height gain as the result of bone elongation is driven by chondrogenesis occurring at the growth plate, which is a cartilaginous structure located near the ends of weight-bearing bones

\footnotetext{
*Correspondence: yueshen@njmu.edu.cn; liuchen@njmu.edu.cn; sycheng@njmu.edu.cn

${ }^{\dagger}$ Qiu-fan Xu and Si-Yang Li contributed equally to this work

${ }^{1}$ Department of Medical Genetics, School of Basic Medical Sciences, Nanjing Medical University, Jiangsu 211166 Nanjing, P. R. China

Full list of author information is available at the end of the article
}

in children [4]. Thus, reduced chondrogenesis at growth plates is the main cause underlying short stature.

The rate of chondrogenesis at growth plates depends on several factors, including nutritional intake, systemic hormonal levels, as well as paracrine growth factors and the extracellular matrix signaling [4-7]. Consequently, genetic lesions that disrupt the regulation of in these systems will result in short stature. In fact, genetic testing of children with abnormal growth has identified multiple intracellular pathways that involved in chondrocyte differentiation in the growth plate. One of them is the RAS oncogene and the mitogen-activated protein kinase (MAPK) signaling pathway, which integrate signals from several growth factors such as growth hormone $(G H)$, fibroblast growth factors $(F G F s)$ and epithelial growth factor $(E G F)$ [8-11]. Mutations in this pathway underpin a number of genetic syndromes that are collectively termed 'rasopathies'; these

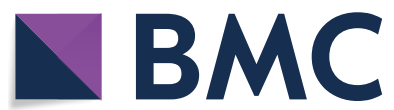

(c) The Author(s) 2021. This article is licensed under a Creative Commons Attribution 4.0 International License, which permits use, sharing, adaptation, distribution and reproduction in any medium or format, as long as you give appropriate credit to the original author(s) and the source, provide a link to the Creative Commons licence, and indicate if changes were made. The images or other third party material in this article are included in the article's Creative Commons licence, unless indicated otherwise in a credit line to the material. If material is not included in the article's Creative Commons licence and your intended use is not permitted by statutory regulation or exceeds the permitted use, you will need to obtain permission directly from the copyright holder. To view a copy of this licence, visit http://creativeco mmons.org/licenses/by/4.0/. The Creative Commons Public Domain Dedication waiver (http://creativecommons.org/publicdomain/ zero/1.0/) applies to the data made available in this article, unless otherwise stated in a credit line to the data. 
include Noonan syndrome, LEOPARD syndrome, Costello syndrome, as well as cardio-facio-cutaneous and neurofibromatosis-Noonan syndrome, a common element of these syndromes is the postnatal growth failure to varying degrees [12].

Since the beginning of the new millennium, mounting evidence has shown that microRNAs and long non-coding RNAs play critical roles in regulating various cellular processes [13]). These non-coding RNAs regulate gene expression and cell signaling through diverse mechanisms. However, while lncRNAs that regulate epigenetic control of gene expression, development as well as govern human traits have been rapidly identified, few lncRNAs have been implicated in skeletal biology. Here, we reported a patient with short stature, hypothalamic dysfunction and mild macrocephaly, who carries a maternally inherited $690 \mathrm{~kb}$ deletion at Chr.1q24.2 encompassing a long noncoding RNA gene, DNM3OS. This lncRNA reading frame is of $7.8 \mathrm{~kb}$ in length embedded on the opposite strand within the 14th intron of the DYNAMIN 3 gene (DNM3), and carries two microRNAs: $m i R-199 a-5 p$ and $m i R-214$. Our data show that it is capable of promoting primary chondrocyte proliferation and inhibiting chondrocyte differentiation via up-regulating nerve growth factor $(N G F)$. Our data further show that the mouse counterpart of this lncRNA, Dnm3os, is a bona fide regulatory factor of skeleton development independent of its embedded co-cistronic miR199a and miR214.

\section{Results}

\section{Deletion of $D N M 3 O S$ is associated with developmental delay}

A young boy was presented to us with clinical manifestation of short stature, hypothalamic dysfunction and mild macrocephaly reminiscent of Noonan syndrome (Fig. 1a, and Additional file 1). The birth weight and height of the proband (II-2) were both below the 5 th percentile among Caucasians, and radiographic examinations first conducted at 2 years of age indicated delayed bone growth; however, laboratory tests found no hormonal imbalance, and tests for mutations in 12 known genes compiled in the Noonan Spectrum Chip were also negative (data not shown). Using oligonucleotide-based array comparative genomic hybridization, we identified a maternally inherited $690 \mathrm{~kb}$ deletion at 1q24.3 (Fig. 1b), which falls within the common chromosomal deletion interval reported in a cohort of 9 patients with facial features, prenatal-onset short stature with delayed bone age, single palmar crease, and brachydactyly similar to the proband [14]. Deletions around 1q24-1q25 have been noted for growth deficiency among a myriad of symptoms [15]. Among 5 protein-coding genes and undefined open reading frames in the deleted region, we were drawn to a transcription unit called DNM3OS on the opposite strand in the 14th intron of DNM3, which encodes no protein but two microRNAs, miR-199a-5p and miR-214 (Fig. 1b). In the literature, homozygous deletion of murine Dnm3os was reported to cause severe skeletal defects in new born mice including cranial deformity, which was most likely the cause of postnatal lethality, but it was not clear whether the long noncoding RNA Dnm3os as a whole, or miR-199a-5p or miR-214 plays that essential role for the skeletal development [16]. Previously we demonstrated that miR-214 downregulates $\mathrm{N}$-ras to promote myogenic differentiation at the expense of osteogenic differentiation [17]; however, genetic ablation of $m i R-214$ did not lead to obvious signs of Noonan syndrome-like features including growth delay in mice $[18,19]$ (Additional file 2: Fig. S1). Nevertheless, expression of 7 Noonan syndrome genes in the Ras pathway was drastically increased (Additional file 3: Fig. S2) in MEF cells isolated from $m i R-214$ knock-out mice, in keeping with the presence of $m i R$ 214 recognition sites in the 3'UTR or coding regions of these RNA transcripts (Additional file 4: Table S1). Using real-time PCR, we found that the levels of DNM3, DNM3OS, and miR-214 RNA transcripts in the peripheral blood of the proband (II-2) and his carrier mother (I-2) were only $50 \%$ of those in his non-carrier brother (II-1) (Fig. 1c-f). Interestingly, the peripheral expression of 4 out of the same 7 Noonan syndrome genes tested in $m i R-214 \mathrm{KO}$ mice was significantly increased in carriers II-2 and I-2 relative to the non-carrier II-1 (Fig. $1 \mathrm{~g}-\mathrm{m}$ ). These data suggest that DNM3OS carries an essential function that accounts for the roles of chromosomal 1q23-1q25 interval in skeletal development and although the ablation of $m i R-214$ in mice is not sufficient to cause phenotype that resembles any aspect of the partial Noonan syndrome manifestation seen in the proband, it nevertheless partakes in the regulation of skeletal growth via the Ras pathway.

(See figure on next page.)

Fig. 1 Deletion of IncRNA-DNM3OS is associated with autosomal dominant inheritance of 1q24 deletion syndrome phenotypes. a Family tree of the proband (II-2). $\mathbf{b}$ Chromosomal maps of a cohort of 9 individuals with 1q24-25 deletions. c- $\mathbf{f}$ RT-qPCR quantifications of DNM3 mRNA, InCRNA-DNM3OS, miR-214, and miR-199 in the blood. The 3'stem-loop primer for human miR-199 recognizes both miR-199a and miR-199b on chromosome 9, the latter of which is not affected by the deletion. g- $\mathbf{m}$ RT-qPCR quantifications of N-RAS, CBL, SHOC2, RAF1, BRAF, SOS1, and PTPN11 in the blood. One-way ANOVA test was used for statistical analysis. ${ }^{* *} \mathrm{P}<0.01,{ }^{* * *} \mathrm{P}<0.001$, and ns, not significant 
a

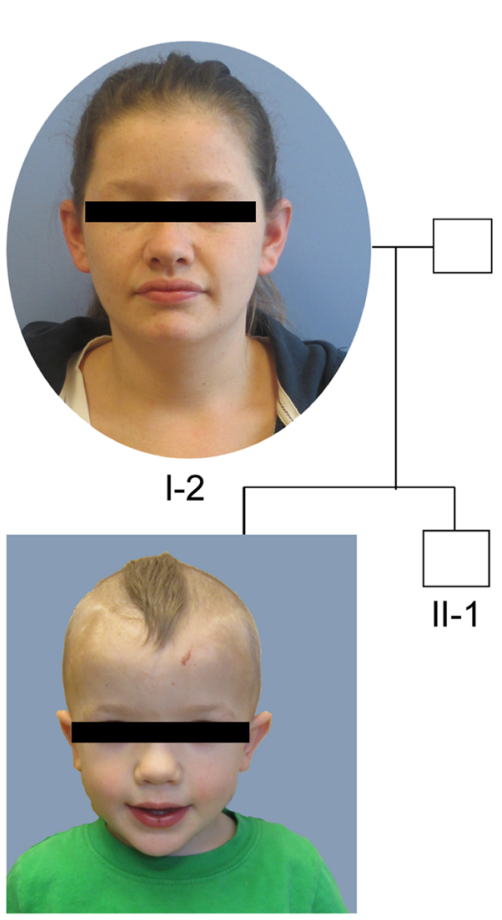

II-2

C

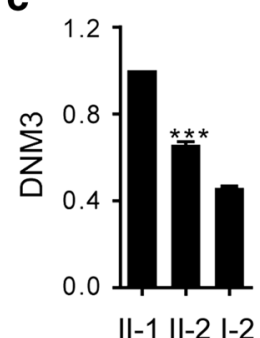

g
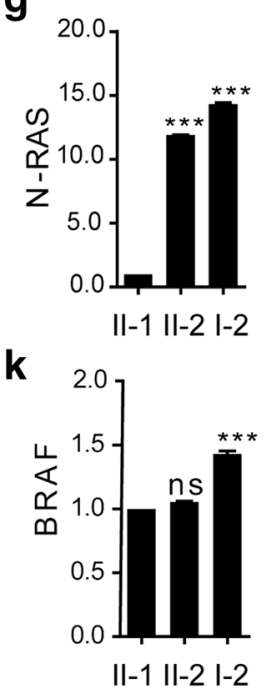

b

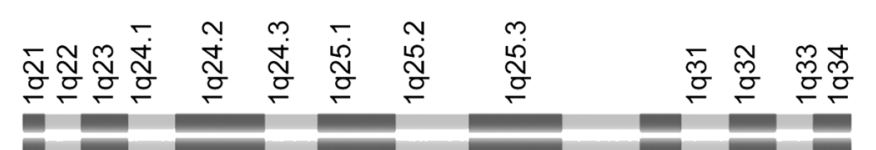

patient
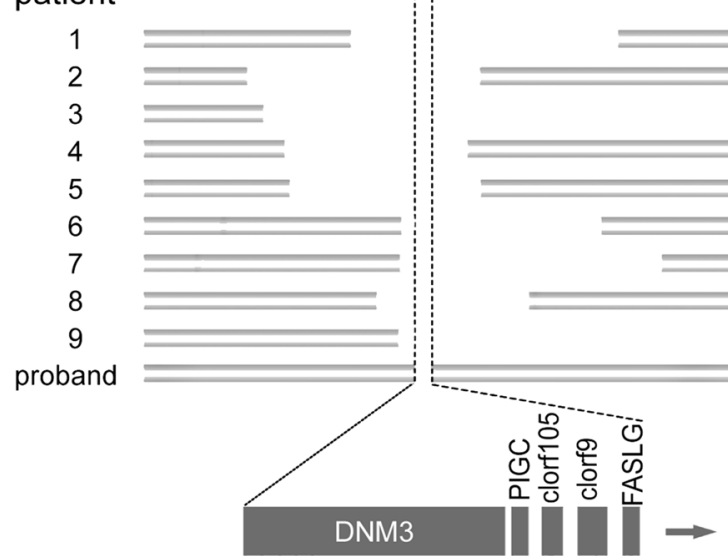

DNM3

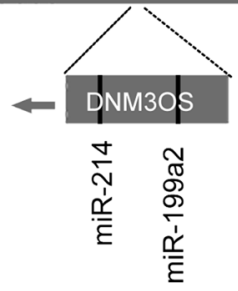

d

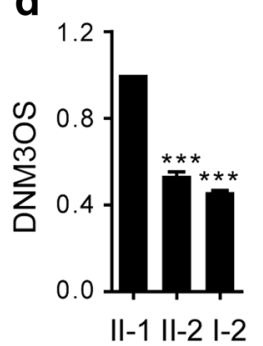

e
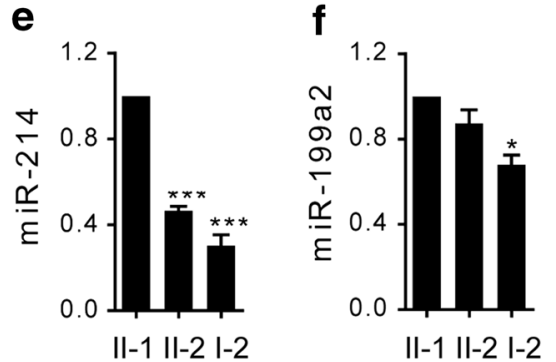

h

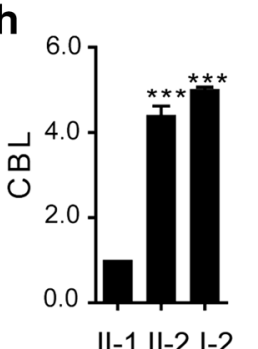

i
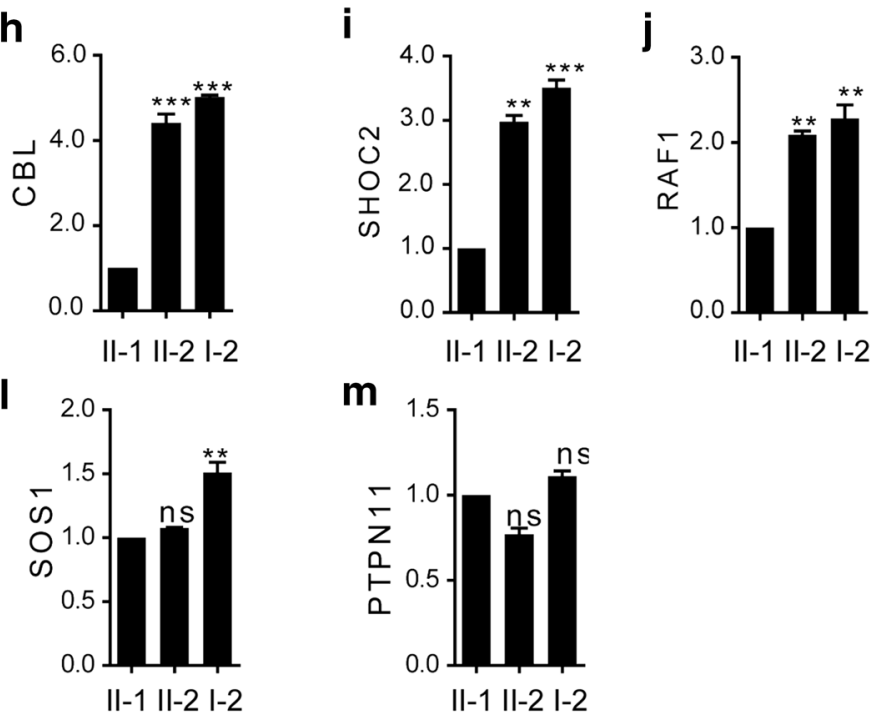

m

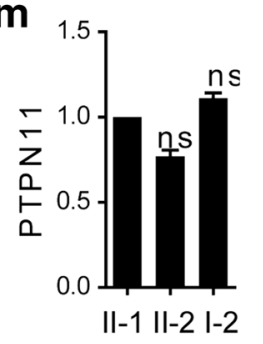




\section{Murine Dnm3os is required for maintaining the proliferative potential of articular chondrocytes}

Both human and mouse Dnm3os encodes a $7.8 \mathrm{~kb}$ RNA transcript, which was identified due to its depleted expression in mouse limb buds lacking basic helixloop-helix transcription factor Twist [20]. First appearing in limb buds and other future skeletal elements [17], robust Dnm3os expression was reported in perichondrial cells and periarticular chondrocytes at the cartilage growth plate [16]. Using RNA-FISH, we found that full length Dnm3os RNA is confined to the nucleus (Fig. 2a). During embryonic development, chondrocytes in the long bone growth plate undergo an orderly proliferation and differentiation process that eventually gives rise to trabecular bones [21]. This chondrogenic process can be faithfully recapitulated in vitro using isolated immature primary mouse articular chondrocytes (iMAC) [22]. We observed that expression of $D n m 3 o s$ and its co-cistronic $m i R-199 a-5 p$ and $m i R$ 214 progressively increased (Fig. 2b, c) following several passages in the maintenance medium as the cells gradually reverted to a "dedifferentiated" state marked by the switch of cell surface collagen types from Col2a1 to Colla1 (Fig. 2d, e). Correspondingly, the expression of chondrogenic transcription factor SOX9 gradually decreases with passaging (Additional file 5: Fig. S3A). When introducing shDnm3os, the switch of cell surface collagen types from Col2a1 to Col1a1 was slightly slowed, suggesting that Dnm3os was not a key fact for dedifferentiated (Additional file 5: Fig. S3B). Conversely, expression levels of these non-coding RNAs all decreased dramatically after the cells were induced to differentiate into hypertrophic chondrocytes marked by Col10a1 and MMP13 (Fig. 2f, g). These spatial and temporal expression patterns of Dnm3os are consistent with an essential role in promoting the proliferation but suppressing the differentiation of chondrocytes. To ascertain such a function, we silenced Dnm3os expression using shRNAs in the iMAC before inducing their differentiation. At the end of two weeks, immunofluorescence microscopy indicated that over $60 \%$ of scrambled shRNA transfected cells (marked by GFP) still retained Col2a1 and Sox9, but these percentages dropped to below 20 and $40 \%$, respectively, in the cells transfected with shDnm3os (Fig. 2h, i, l, m). Remarkably, many GFP positive cells that received $\mathrm{sh} D n m 3 o s$ completely lost Col2a1 and Sox9 (Fig. 2h, i, l, m), indicating that they had differentiated further into hypertrophic chondrocytes (Fig. 2b). Elevated expression of Col10a and Mmp13 confirmed the role of silencing Dnm3os in promoting chondrogenic differentiation (Fig. 2j, k, n, o). The expression level of Dnm3os maintained at a relative low level in the cells transfected with shDnm3os (Fig. 2p). Moreover, EdU assay showed Dnm3os silencing decreased the proliferation of iMAC by $10 \%$ (Additional file 5: Fig. S3 C-E).

In addition to controlling Ras pathway genes (Additional file 3: Fig. S2), forced expression of $m i R-214$ and miR-199a blocked chondrogenesis (Additional file 6: Fig. S4A-F) and promoted proliferation (Additional file 6: Fig. S4G-H). Thus, to determine if the large Dnm3os is sufficient in regulating chondrogenesis or requires the two co-cistronic microRNAs, we cloned the mouse full length Dnm3os (FL) and generated a mutant that lacks both microRNAs (DKO). RT-QPCR analysis confirmed the overexpression of FL and DKO in iMAC cells (Additional file 5: Fig. S3H). Forced expression of either FL or the DKO mutant Dnm3os impeded the chondrogenesis as evident by reduced staining by alcian blue (Fig. 3a, b) or of alkaline phosphatase (Fig. 3c, d), which mark the cartilage matrix proteins and the mature hypertrophic chondrocytes, respectively. The effects of DKO in chondrogenesis were not due to the dominant-negative inhibition of endogenous Dnm3os function, since overexpression of DKO inhibited chondrogenesis as shown by decreased Mmp13and Colloalin iMAC cells silencing endogenous Dnm3os with shDnm3os transfection (Additional file 5: Fig. S3F-G). Forced expression of the DKO mutant also showed similar propensity to down-regulate the same cohort of Ras pathway genes as the parental RNA (Additional file 7: Fig. S5), indicating that Dnm3os can function independently of the two microRNAs. Finally, to ascertain if Dnm3os directly promotes the proliferation of articular chondrocytes, we labeled the cells with EdU and found that both the FL and DKO mutant Dnm3os accelerated the cell growth (Fig. 3e, f).

\footnotetext{
(See figure on next page.)

Fig. 2 LncRNA-Dnm3os is required for maintaining the proliferative potential of chondrocytes. a RNA-FISH detection of IncRNA-Dnm3os in NIH3T3 cells using Quasar 570 labelled Stellaris oligonucleotide probe. b Flow chart of in vitro chondrogenic differentiation. $\mathbf{c}$ RT-qPCR detection of RNAs in primary articular chondrocytes at passages as noted. $\mathbf{d}$, e RT-qPCR detection of proliferating chondrocyte marker, Col2a1, and osteoblast marker Col1a1, respectively; the latter cell descends from mesenchymal lineage. $\mathbf{f}$ RT-qPCR detection of RNA and $\mathbf{g}$ hypertrophic chondrocyte markers Col10a and Mmp13 following differentiation for 2 weeks. h-k IF staining of Col2a1, Sox9, Col10a1, and Mmp13 and I-o quantification thereof following differentiation for 2 weeks. Prior to induction of differentiation, the primary articular chondrocytes were transfected with scrambled shRNA (ctrl) or shDnm3os, both of which carried a GFP marker. $\mathbf{p}$ RT-qPCR detection of Dnm3os at the start and end of in vitro chondrogenic differentiation. Student T-test was used for statistical analysis. ${ }^{* *} \mathrm{P}<0.01,{ }^{* * * P}<0.001$
} 
a

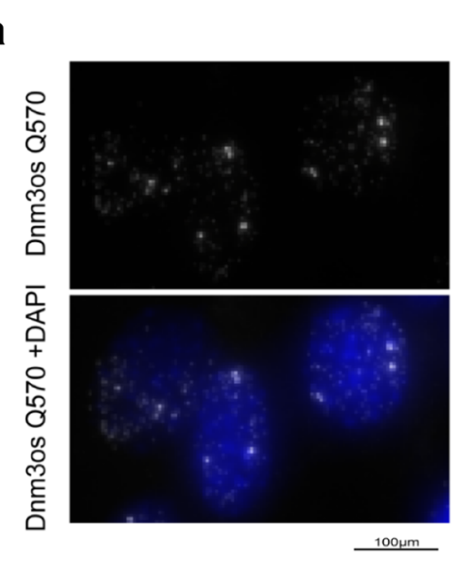

b
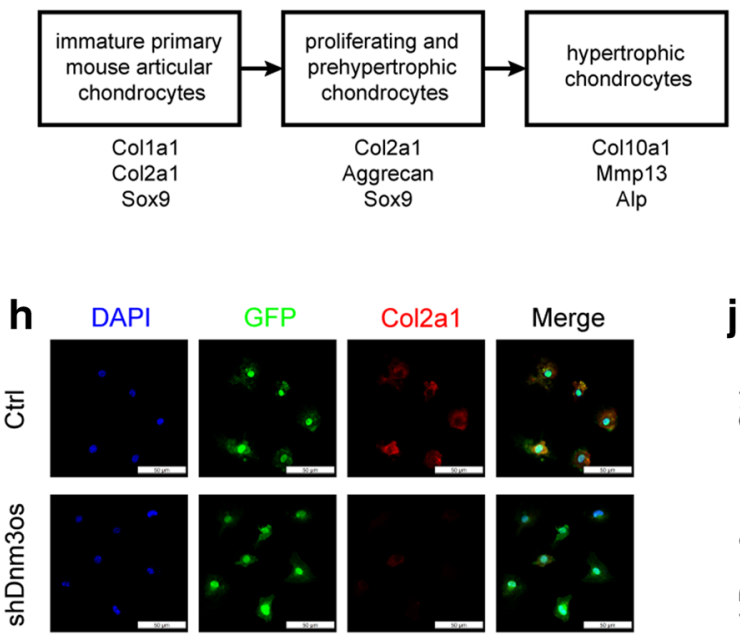

i DAPI
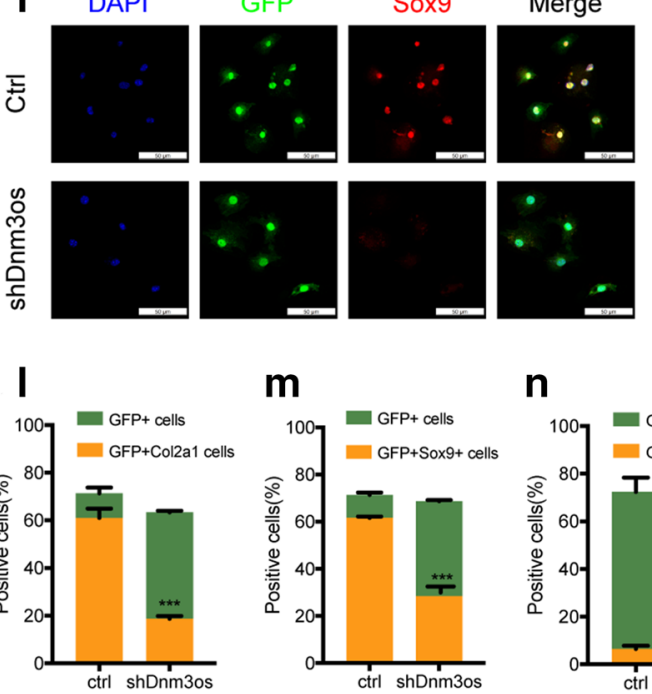

n

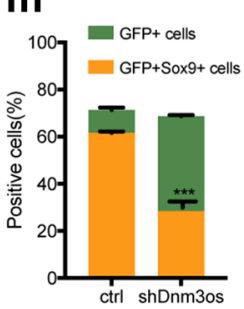

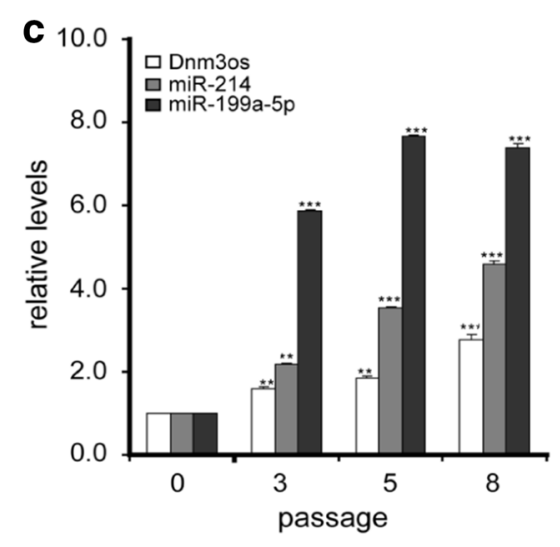

d
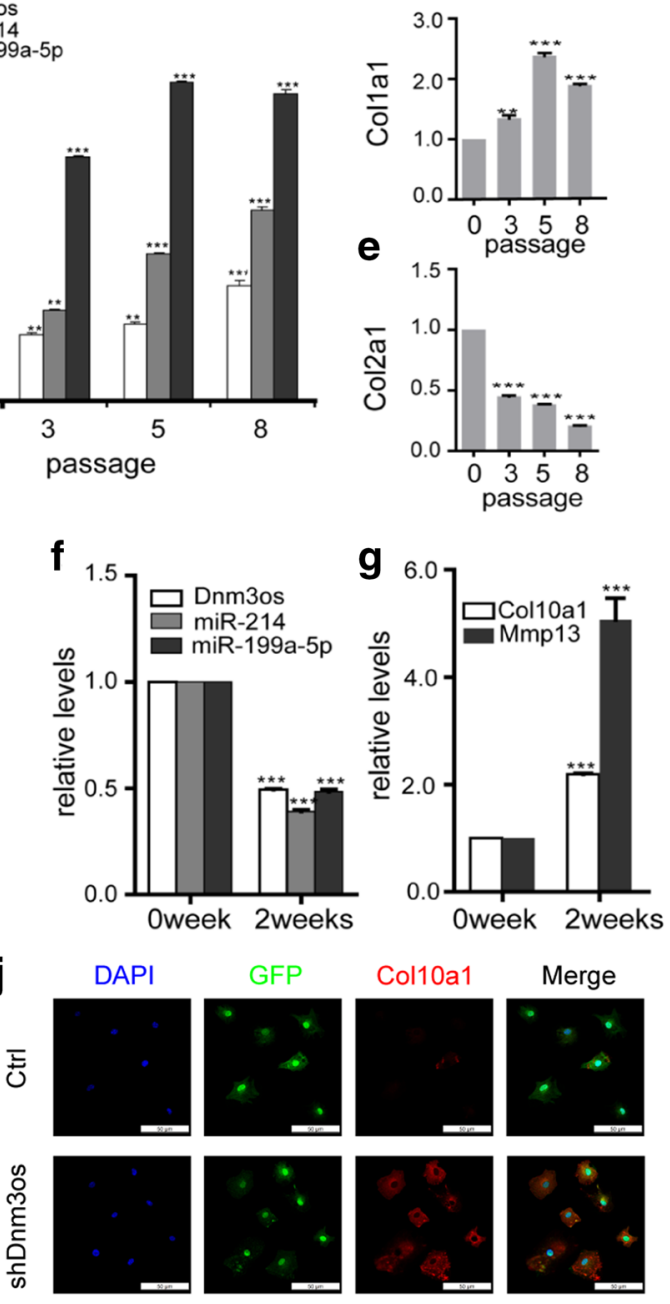

k
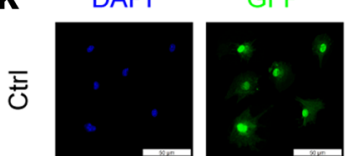

Mmp13
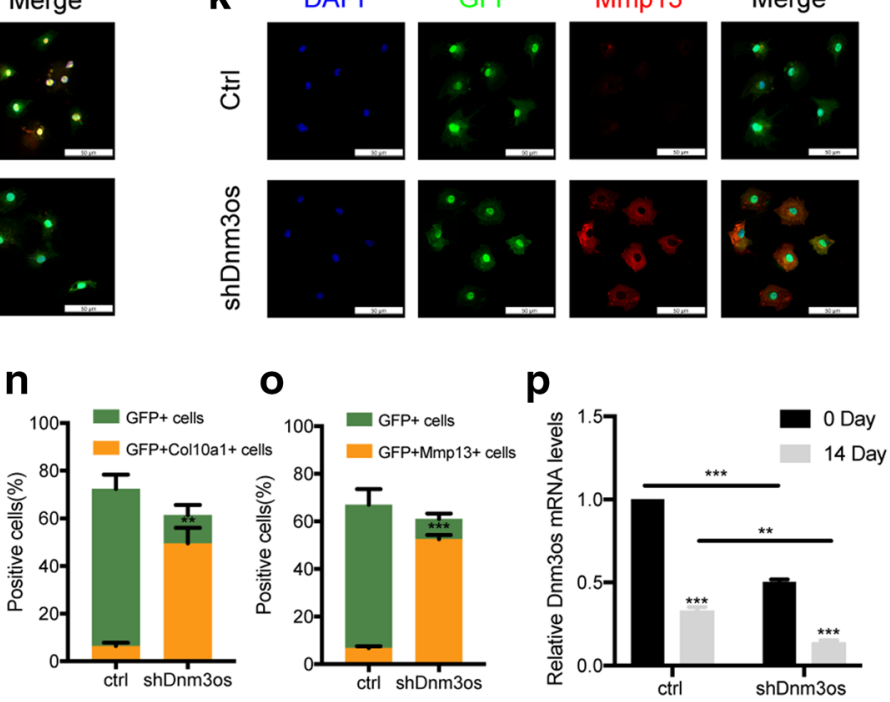


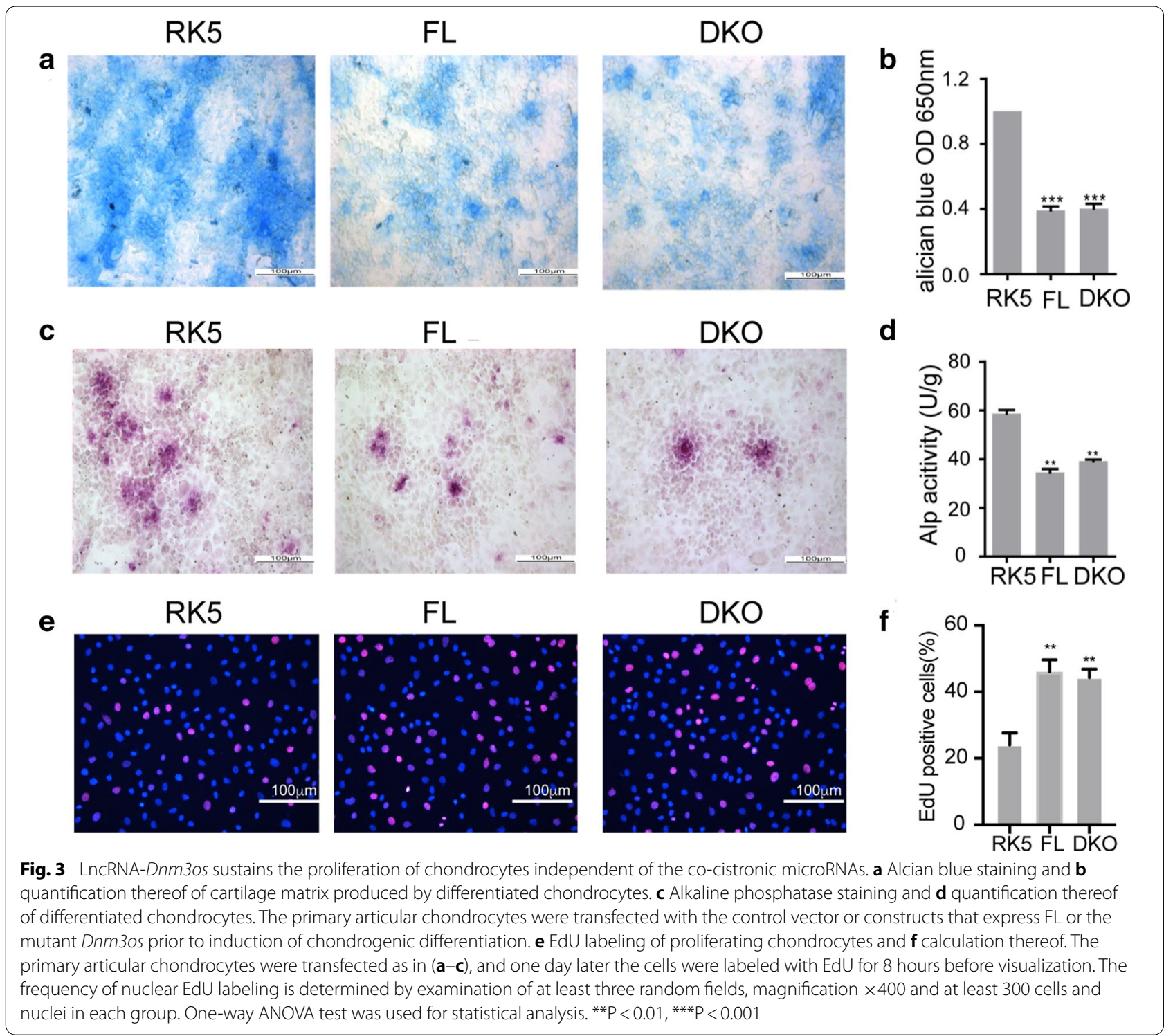

\section{Dnm3os is required for maintaining the proliferative potential of ATDC5 cells}

To corroborate the above observation in primary mouse articular chondrocytes, we took the advantage of ATDC5 cells that are widely used as an in vitro model for chondrocyte differentiation to test the pro-proliferative role of Dnm3os. Alcian blue staining images collected by light microscopy over an 18-day time-course indicated that insulin-supplemented differentiation medium cultivation successfully induced the differentiation of ATDC5 cells to mature hypertrophic chondrocytes (Additional file 8: Fig. S6A). Decreased Col2a1 and increased Col1a1 expression confirmed the differentiation on the biomarker (Additional file 8: Fig. S6B, C). Consistent with primary mouse articular chondrocytes, the expression of Dnm3os in ATDC5 cells gradually decreased with the chondrogenic differentiation (Additional file 8: Fig. S6D). Overexpression of FL and the DKO mutant Dnm3os activated ATDC5 cells proliferation and impeded the chondrogenesis as evident by 2-fold increased EDU positive cells (Fig. 4a, b and Additional file 5: Fig. S3I) and absent Alcian blue staining (Fig. 4c), while silencing Dnm3os with shRNA inhibited the proliferation of ATDC5 cells (Fig. 4d, e). We further employed RT-QPCR to examined the expression of Sox9 in FL and DKO transfected ATDC5 cells and iMAC cells. Both overexpression of FL and DKO increased the expression level of Sox9 in ATDC5 cells, while the effect in iMAC cells were barely observed (Additional file 8: Fig. S6E, F). However, the expression of Sox9 decreased with iMAC cells passaging 
a

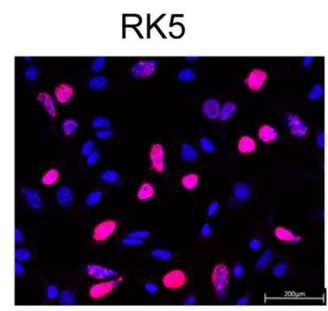

DAPI EdU
$\mathrm{FL}$

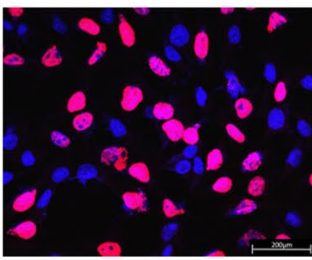

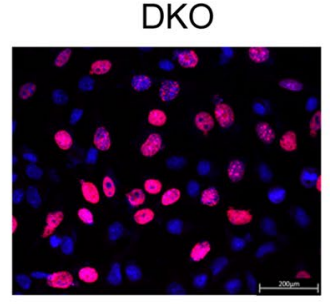

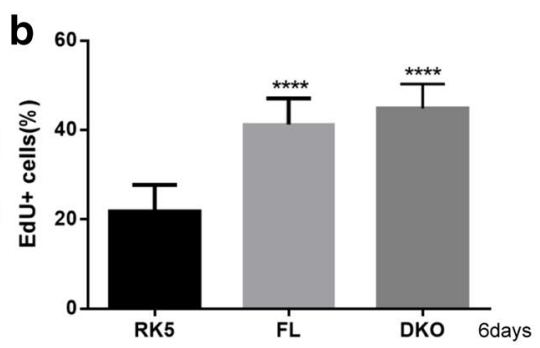

C

RK5

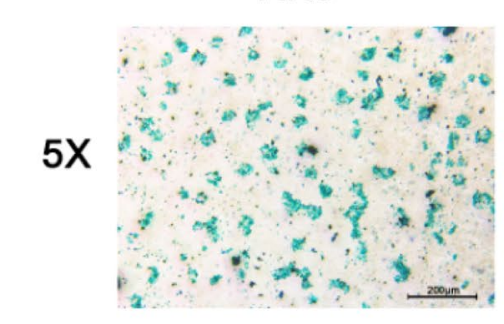

$20 x$

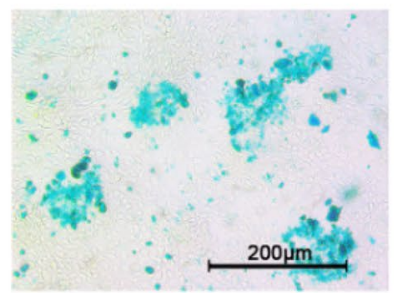

FL
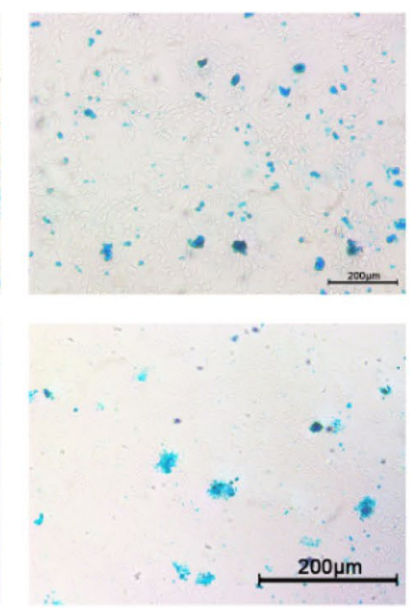

d

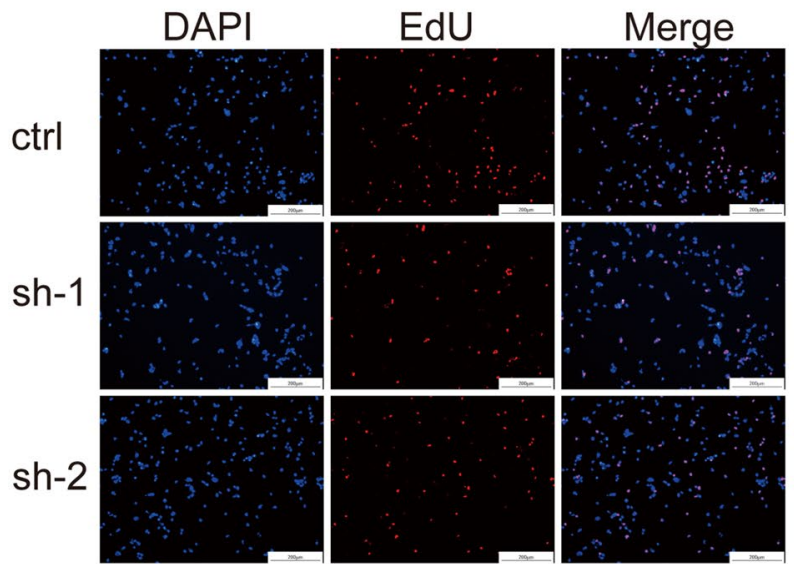

e

DKO
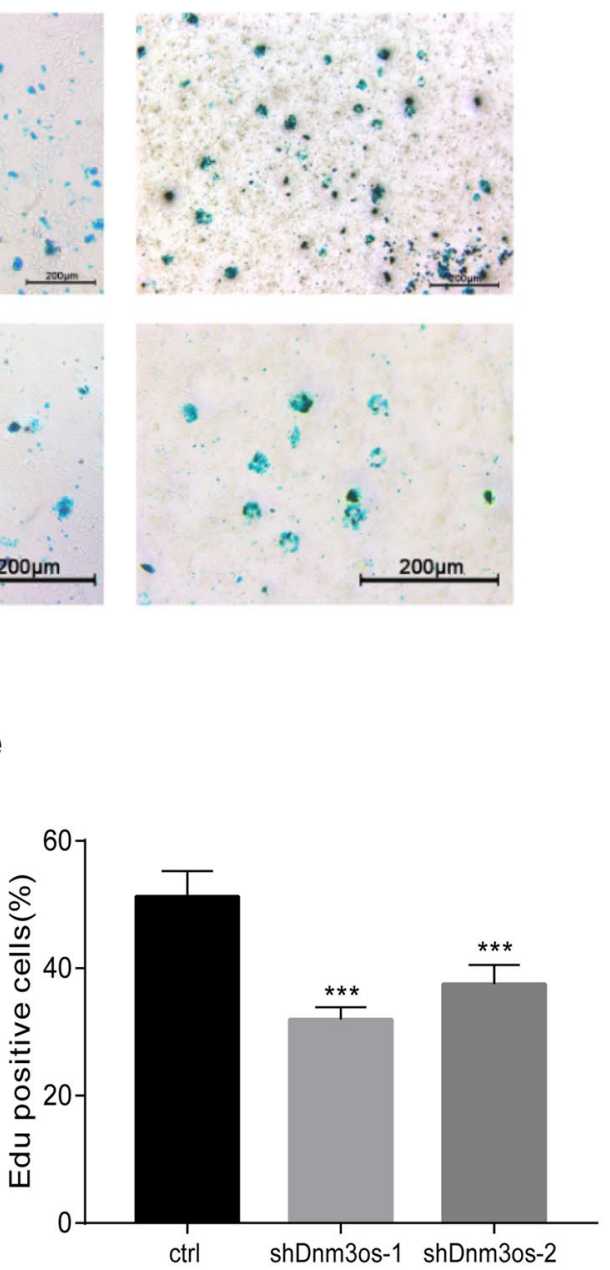

Fig. 4 LnCRNA-Dnm3os is required for maintaining the proliferative potential of the ATDC5 cells. a EdU labeling of proliferating ATDC5 cells and $\mathbf{b}$ calculation thereof. The ATDC5 cells were transfected by RK5, FL and DKO individually, and one day later the cells were labeled with EdU for 8 hours before visualization. $\mathbf{c}$ Alcian blue staining of cartilage matrix produced by differentiated ATDC 5 cells. $\mathbf{d}$ EdU labeling of proliferating ATDC5 cells and e calculation thereof. The ATDC5 cells were transfected with scrambled shRNA, shDnm3os-1 and shDnm3os-2 individually, and one day later the cells were labeled with EdU for $8 \mathrm{~h}$ before visualization. The frequency of nuclear EdU labeling in (a) and (d) were determined by examination of at least three random fields, magnification $\times 400$ and at least 300 cells and nuclei in each group. One-way ANOVA test was used for statistical analysis. ${ }^{* * *} \mathrm{P}<0.001$, ${ }^{* * *} \mathrm{P}<0.0001$, and $\mathrm{ns}$, not significant 
(Additional file 5: Fig. S3A), which suggested the overexpression of FL and DKO to some extent counteracted the reduction of Sox 9 caused by passage. These data suggest that Dnm3os contributes to the maintenance of ATDC5 and iMAC cells as chondrogenic progenitors and plays an independent role in regulating chondrogenesis instead of simply acting as a precursor of miRNAs.

\section{NGF is a potential Dnm3os-regulated gene in chondrocytes}

To substantiate the direct link between Dnm3os and chondrogenesis, we performed RNA-seq in FL and DKO mutant Dnm3os transfected primary mouse articular chondrocytes with 2 biological replicates. The result showed 250 and 211 differentially expressed genes in FL and DKO overexpressed cells relative to the RK5 transfected control cells, respectively (Fig. 5a), and of these, 155 were commonly expressed in those two cells transfected with Dnm3os vectors, suggesting that this lncRNA functions as an independent regulator of chondrocytes (Fig. 5a) and DKO acts as a regulator at the transcriptional level. To predict the biological function of Dnm3os, we categorized these 155 genes into defined pathways and we found cell cycle appeared in the top altered pathways, which confirmed the proliferation effect of Dnm3os (Additional file 8: Fig. S6G). To further provide biological insight to the differential expression genes, the 155 genes were assigned into defined pathways by using Reactome databases (Fig. 5b). Among the top 20 significantly altered pathways ranked in the dot plot enrichment map, signal transduction, metabolism and extracellular matrix organization, whose dynamics are key to tissue morphogenesis appeared with majority gene enrichment and statistical significance. We further dissected 8 of the top enriched pathways and found matrix metalloproteinase 3 , collagen genes including Colsa2, Col23a and growth factors including $N G F, F G F$, as visualized by the heat map (Fig. 5c). The expression changes of these genes suggested the ongoing extracellular matrix remodeling and accelerated cell proliferation. Subsequently, we picked up 14 genes which have been reported to play a role in chondrocyte proliferation or differentiation and verified the change by QPCR analysis (Fig. 5d, e). To exclude the $m i R$ 214 target genes, we detected the expression of these 14 genes in miR-214 transfected ATDC5 cells simultaneously, and found NGF was a potential target gene regulated by lncRNA-Dnm3os specifically (Fig. 5e).

\section{Dnm3os-induced NGF maintains the proliferative potential of ATDC5 cells}

As a neuropeptide, NGF was involved in cartilage metabolism and is reported to mediate the chondrogenic differentiation of mesenchymal stem cells [23]. To determine if NGF play a role in Dnm3os mediated chondrocytes proliferation, we treat FL, DKO mutant and $m i R-214$ transfected ATDC5 cells with PD90780, an inhibitor of NGF. 24 hours after treatment, we labeled the cells with EdU and found that inhibition of NGF blocked FL and DKO mutant Dnm3os-induced chondrocytes proliferation (Fig. 6a, b). On the other hand, the chondrogenesis was promoted by PD90780 as evident by increased staining by alcian blue in FL and DKO mutant Dnm3os transfected cells after 18-days differentiation (Fig. 6c, d). Consistently, FL and DKO mutant Dnm3os-induced collagen types switch from Col1a1 to Col2a1 was interrupted by PD90780 (Fig. 6e, f). MMP9, a target gene of NGF was downregulated with treatment of PD90780 and validated the inhibitory effect of the compound (Fig. 6g). RT-QPCR analysis of Col10a1 confirmed the that inhibition of NGF in FL and DKO constructs overexpressing cells promoted differentiation (Fig. 6h). These data collectively indicated these chondrocytes have been released from the bondage of Dnm3os and entered the differentiation state.

\section{Discussion}

Skeletal development is a complex process that exquisitely controlled both spatially and temporally by cell signaling networks and gene regulation programs. In the past decades, studies of congenital human disease reveal a great deal of genes that involved in bone growth including Ras-MAPK pathway, Wnt and Hedgehog signaling [24, 25]. However, the contribution of non-coding RNA especially lncRNA to skeletal development still remains unclear. Here, we present evidence that shows LncRNA-Dnm3os is required for maintaining the proliferative potential of articular chondrocytes, and we demonstrate that Dnm3os has specific gene

\footnotetext{
(See figure on next page.)

Fig. 5 RNA-sequencing analysis of LncRNA-Dnm3os regulated genes in chondrogenesis. a Quantitative RNA-seq comparison of differentially expressed genes in FL and DKO transfected primary articular chondrocytes. Total number of genes with at least 1.15-log2Fold Change are indicated. b Heat map representation of the top 8 enriched pathway with highest fold change in FL and DKO transfected primary articular chondrocytes. c Top 20 enriched Reactome pathways in FL and DKO transfected primary articular chondrocytes. The size and color of the dots represent the enriched gene number and the range of $\mathrm{p}$ values, respectively. $\mathbf{d}$ Genes associated with chondrogenesis that are up- or down-regulated in FL and DKO transfected primary articular chondrocytes. e Verification of LnCRNA-Dnm3os regulated genes in (d) by RT-qPCR in chondrocytes transfected by RK5, FL, DKO and miR214 individually. Student T-test was used for statistical analysis. ${ }^{* P}<0.1$ and ${ }^{* *} \mathrm{P}<0.01$
} 
a

\begin{tabular}{cccc}
\multicolumn{3}{c}{ Chondrocytes } \\
\multicolumn{2}{c|}{ FL } & \multicolumn{2}{c}{ DKO } \\
250 & 155 & 211
\end{tabular}

b
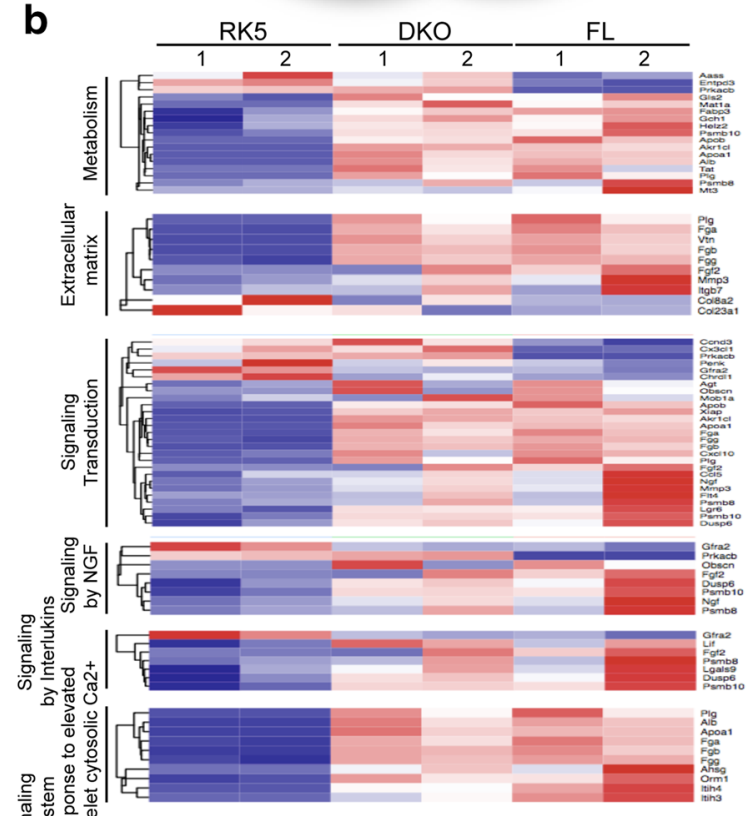

要
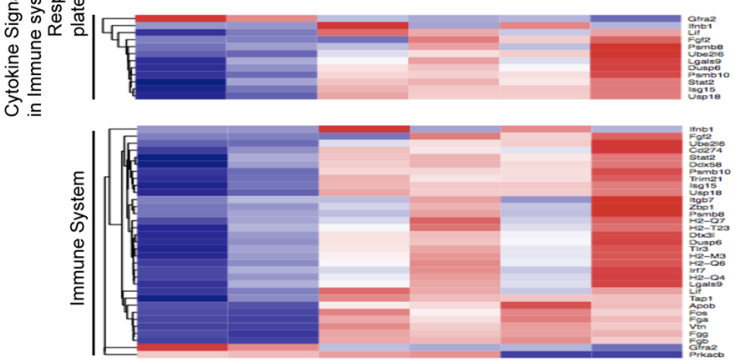

C

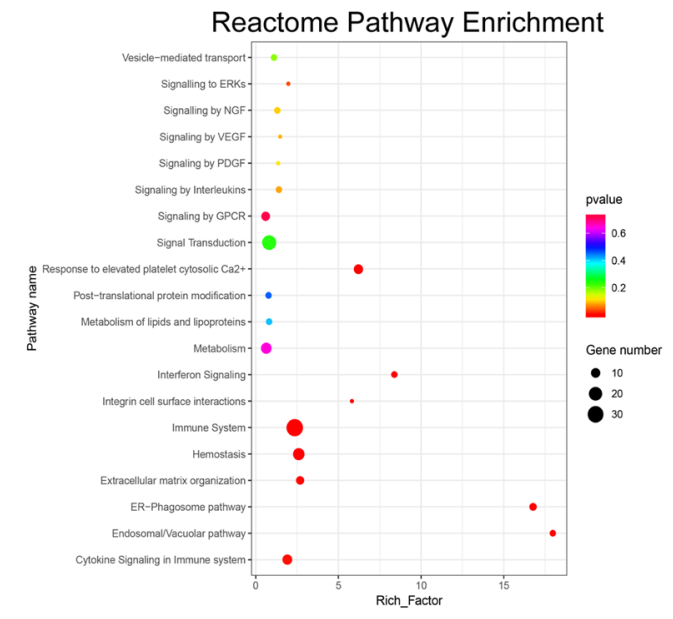

d

Genes Related with Chondrogenesis

\begin{tabular}{|c|c|c|c|}
\hline & GENE & $\begin{array}{c}\text { log2FoldChange } \\
\text { (FL vs RK5) }\end{array}$ & $\begin{array}{c}\text { log2FoldChange } \\
\text { (DKO vs RK5) }\end{array}$ \\
\hline DOWN & SFRP2 & 1.72549336587 & 1.13522006817 \\
\hline UP & IFI35 & 1.18190163 & 1.214087 \\
\hline UP & IFI47 & 2.6299492 & 3.248152 \\
\hline UP & IFIT1 & 1.83942662 & 1.918009 \\
\hline UP & IFIH1 & 1.53234626 & 1.666409 \\
\hline UP & STAT1 & 1.422793 & 1.350369 \\
\hline UP & RSAD2 & 3.380561 & 3.312948 \\
\hline UP & NMI & 1.244962478 & 1.271796 \\
\hline UP & NGF & 1.400348483 & 1.952241 \\
\hline UP & MMP3 & 1.937838 & 2.549021 \\
\hline UP & LIF & 1.611222 & 1.166628 \\
\hline UP & FLT4 & 1.549871 & 2.134068 \\
\hline UP & CXCL10 & 2.515588 & 2.904482 \\
\hline
\end{tabular}

e

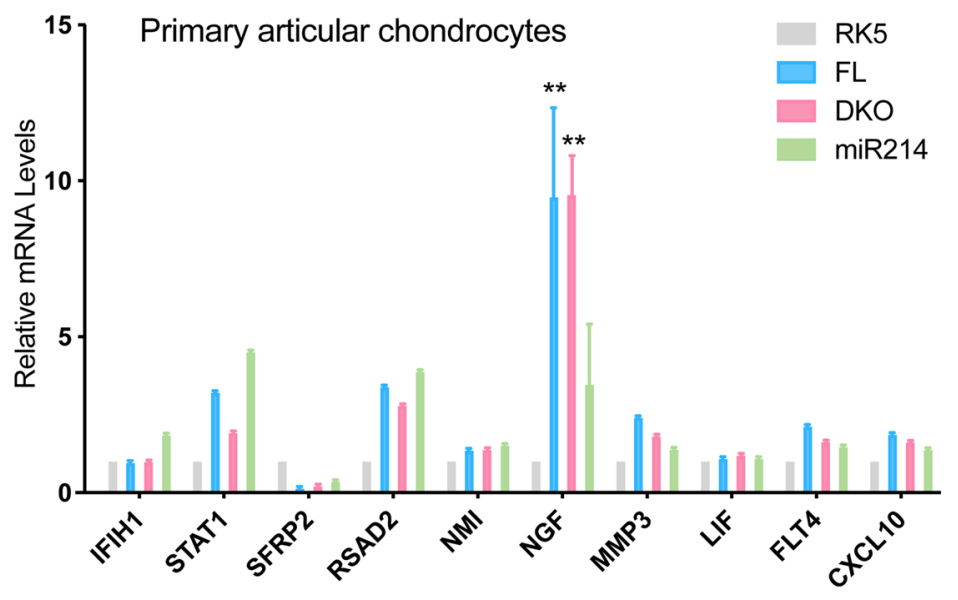


targets such as $N G F$, which impeded chondrocytes to differentiation state. Thus, Dnm3os defines a new class of lncRNAs that serve as transcriptional regulator in addition to produce microRNAs, thereby forming a regulatory network that maintains a proper pool of proliferating chondrocytes to supply bone growth through endochondral ossification, which account for the short stature.

In 2011, Burkardt et al. reported nine patients with a core clinical symptom of mental retardation, microcephaly accompanied by short stature, and identified a crucial deletion region spanning $1.9 \mathrm{Mb}$ at $1 \mathrm{q} 24.3 \mathrm{q} 25.1$ [14]. The deleted region contains 13 genes including Dynamin 3 (DNM3) and CENPL, which encodes a protein essential for centromeric function, mitotic progression and synaptic reaction. Later, Ashraf $\mathrm{T}$ described 2 patients with 1q24 microdeletions and the skeletal phenotype, but had normal intellect or mild learning impairment [26]. Genetic testing of these 2 patients narrows the skeletal abnormalities to a region containing only $D N M 3$ and a transcript union in the opposite strand of DNM3, which called DNM3 opposite strand (Dnm3os). This unit can be transcribed into a long non-coding RNA Dnm3os (LncRNA-Dnm3os), which was described as a precursor of two microRNAs: $m i R-199 a-5 p$ and $m i R-214$. It was reported that Dnm3os, miR214 and miR199a-5p are abundant in the skeleton system containing limb and skull [27]. Dnm3os deletion mice exhibited several skeletal abnormalities, including craniofacial hypoplasia and defects of dorsal neural arches [16]. Down regulation of miR199a-214 cluster, especially $m i R-214$ was considered to be responsible of the phenotype. While for quite a long time, LncRNA-Dnm3os was described as a precursor of these two miRNAs. However, miR-214 KO mice were born at Mendelian ratios and displayed a minimal reduction in body weight compared with WT littermates. Our experiments revealed scientific explanations for these paradoxical phenomena. First, LncRNA-Dnm3os may have compensatory effect of skeletal development since LncRNA-Dnm3os shares most of the target genes with $m i R-214$, and genetic deletion of the miR199a-214 cluster did not abolish the regulatory effect of LncRNADnm3os (Figs. 3 and 4). The other possible explanation is LncRNA-Dnm3os has its own target genes which involved in skeletal development. Thus, the Dnm3os transcript unit defines a regulatory network between lncRNA and miRNAs.

During the past decades, emerging evidence suggests that lncRNAs can play a crucial role in manipulating various cellular processes. In particular, lncRNAs can serve as mater gene regulators at transcriptional and posttranscriptional levels,participating in embryonic development and occurrence of diseases. In some cases, lncRNAs can act as baits of microRNA and sequestrate microRNA for target mRNAs transcriptional repression. Other lncRNAs including BACE1 AS regulate gene expression by competing with miRNAs. And for some lncRNAs, their degradation can be triggered by microRNA. Recently, research works defined the interaction between nucleolin, ILF-2 and IncRNA-Dnm3os by RNA pull-down assays with macrophage nuclear lysates, which indicates that lncRNA-Dnm3os is more than a precursor of microRNAs. Due to the obvious and direct link to vertebrate skeleton development, lncRNA-Dnm3os may also have unique function in this biological process.

\section{Conclusions}

This study demonstrated that lncRNA-DNM3OS maintains the proliferation and restrains premature differentiation of chondrocytes independent of the co-cistronic microRNAs miR-199a and miR-214. In addition, mechanistic studies showed NGF as a key target of lncRNADNM3OS that supports chondrocyte proliferation. Combined with our findings, IncRNA-DNM3OS likely plays an important role in regulating skeletal development by triggering NGF signaling. Future studies are required to ascertain whether there are more particular genes or signaling pathways regulated by lncRNA-DNM3OS.

\section{Materials and methods Single family case studies}

The studies of this case obtained the informed consent from all subjects. For publication of photos, consent was obtained from the patients.

\footnotetext{
(See figure on next page.)

Fig. 6 LncRNA-Dnm3os sustains the proliferation of chondrocytes by upregulating NGF. a EdU labeling of proliferating Atdc5 cells and $\mathbf{b}$ calculation thereof. The ATDC5 cells transfected by RK5, FL, DKO, and miR214 were treated with vehicle of PD90780 (1\%o DMSO) or PD90780 $(10 \mathrm{ug} / 1 \mathrm{ml})$ for 24 hours, and one day later the cells were labeled with EdU for $8 \mathrm{~h}$ before visualization. The frequency of nuclear EdU labeling is determined by examination of at least three random fields, magnification $\times 400$ and at least 300 cells and nuclei in each group. c Alcian blue staining and $\mathbf{d}$ quantification thereof of cartilage matrix produced by the differentiated ATDC 5 cells. The ATDC5 cells were transfected and treated with PD90780 as in (a). e-h RT-qPCR quantifications of Col1a1, Col2a1, Mmp9 and Col10a1 in ATDC5 cells transfected and treated with PD90780 as in (A). Student T-test was used for statistical analysis. ${ }^{*} \mathrm{P}<0.1,{ }^{* *} \mathrm{P}<0.01,{ }^{* * *} \mathrm{P}<0.001$, and ns, not significant
} 

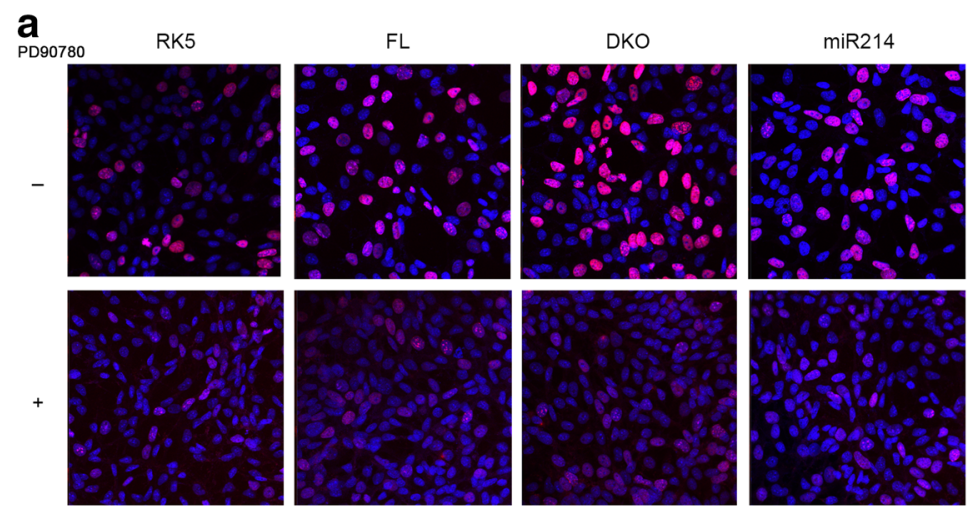

b
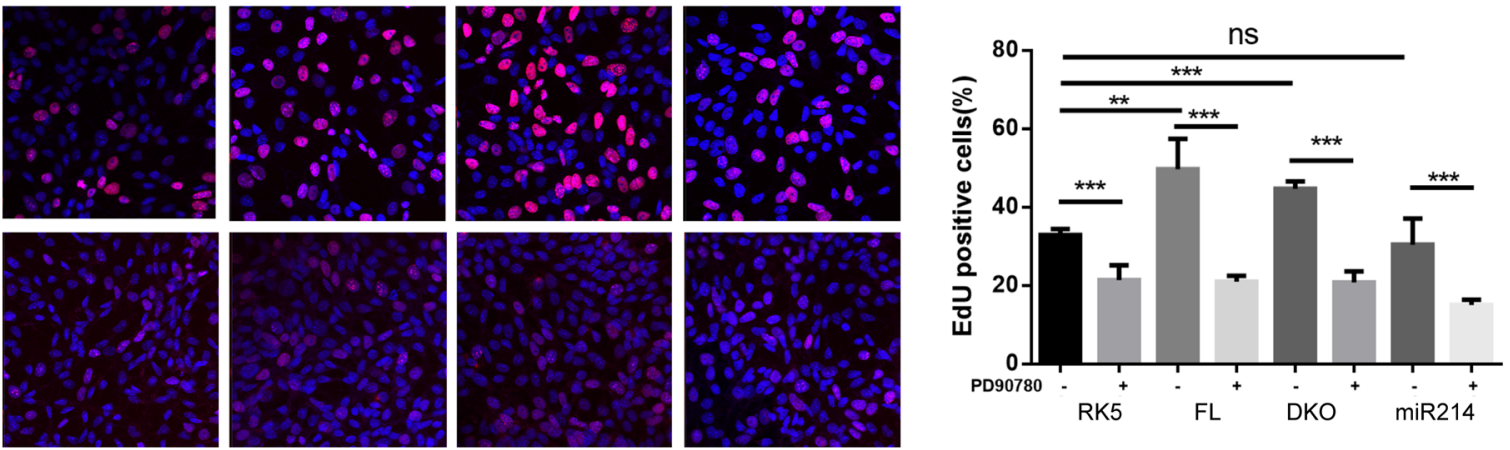

C

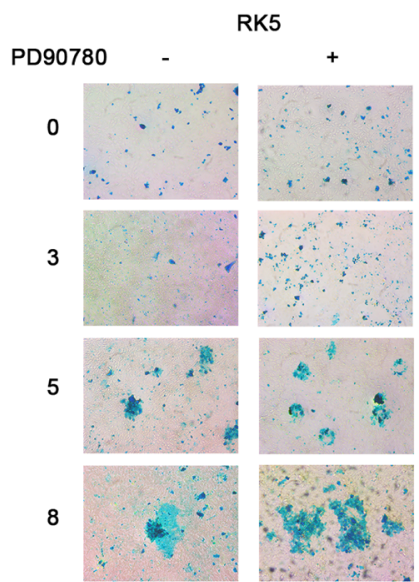

FL

DKO

miR214

d
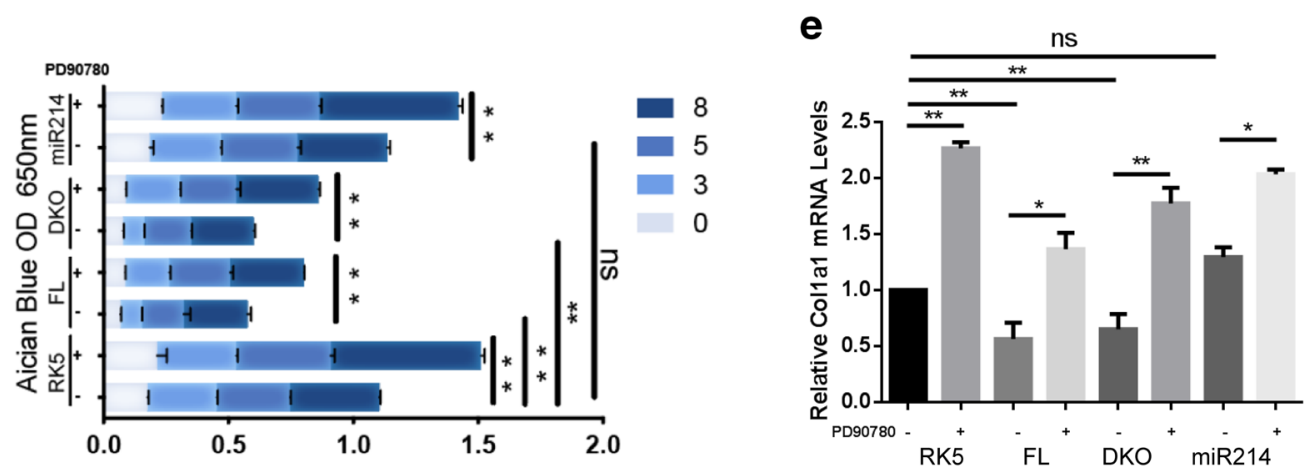

f

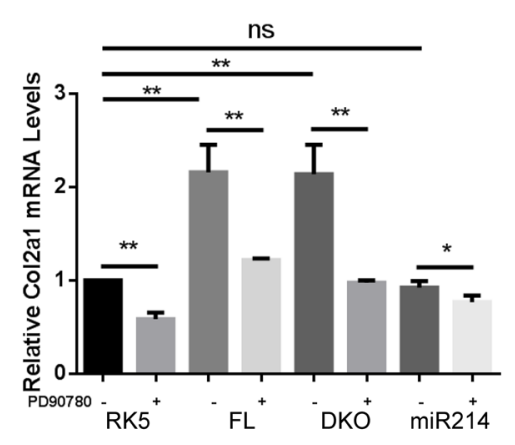

g

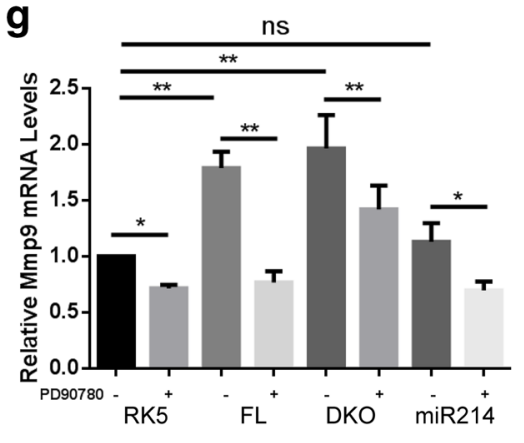

h

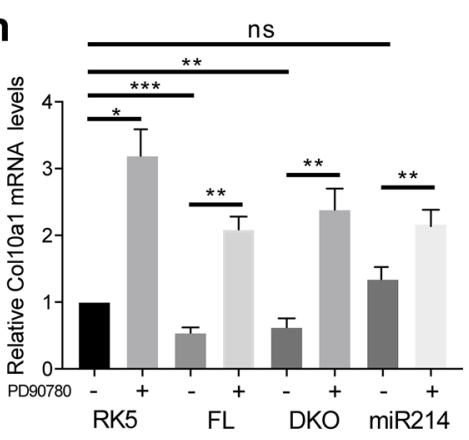




\section{Animals}

The studies with animals follow the guidelines and ethical regulations. The research program and study protocols were approved by Animal Care and Ethical Committee of Nanjing Medical University (approval number 14,030,111).

\section{Isolation of mouse articular chondrocytes and culture}

Primary chondrocytes were isolated from 5 to 6 days old mice as described. Briefly, cartilages from tibial plateaus and femoral condyles were excised and all extraneous soft tissues were removed. To isolate chondrocytes, the cleaned cartilages were digested two consecutive times in cell culture medium with $3 \mathrm{mg} / \mathrm{ml}$ collagenase D (Roche, Indianapolis, 11088858001 ) at $37{ }^{\circ} \mathrm{C}$ for $45 \mathrm{~min}$, and then overnight with $0.5 \mathrm{mg} / \mathrm{ml}$ collagenase $\mathrm{D}$. The next day, the dislodged cells were passed through a $2 \mathrm{ml}$ Pasteur pipet successively to disperse aggregates, then through a sterile $48-\mu \mathrm{m}$ mesh before collected by centrifugation for $10 \mathrm{~min}$ at $400 \times g, 20^{\circ} \mathrm{C}$. The cells were plated out at a density of $25 \times 10^{3} / \mathrm{cm}^{2}$. Chondrogenic differentiation was induced by replacing culture medium with DMEM/ F12 (1:1) (Gibco,C11330500BT), supplemented with 10\% FBS, $1 \%$ pen\&strep (Gibco, 15140122), $10 \mu \mathrm{g} / \mathrm{ml}$ insulin, $10 \mu \mathrm{g} / \mathrm{ml}$ transferrin, and $3 \times 10^{-8} \mathrm{M}$ sodium selenite (Sigma, I3146). The differentiation medium was changed every 3 days.

\section{Plasmids construction and cell transfection}

The DNA fragment containing full length Dnm3os sequence was amplified by PCR from mouse genomic DNA and inserted between the BamHI and Sall sites in the pRK5 vector to generate pRK5-Dnm3os. PCR-based deletion strategy was used to generate the miR-199a-5p and $m i R-214$ deletion mutant (DKO). The PCR primers used are as follows.

\section{FL-F:TTCCTGGTCCTAAATTCATTGCCAG \\ FL-R:ATAGGAATAAAAATTACAAGTATGAA \\ MiR-199a2D-F1: TTCCTGGTCCTAAATTCATTG CCAG \\ MiR-199a2-R1:ACAGGATTTTCCACACACCGA MiR-199a2D-F2:ACGCCATGGACGGCTGGGGAC} ACA

MiR-199a2D-R2: ATAGGAATAAAAATTACAAGT ATGAA

MiR-214D-R1: AACCTGAAGGACCCAAG

MiR-214D-F1:AAAACCTACCCGAAGTAAAG

$\mathrm{Sh} D n m 3 o s$ was designed by using online tools available from . Oligonucleotides (listed in Additional file 9: Table S2) of $\mathrm{shDnm3os}$ were cloned into pRetroH1G shRNA expression vector. ShRNA with the sense sequence $5^{\prime}$-agatctTTAGTATAGATAATATTT
CtacctgacccataGAAATATTAT CTATACTATTTTTggtacc- $3^{\prime}$ which lacks complementary sequences in the human genome, was used as control (scrambled shRNA).

Primary articular chondrocytes were transfected with endotoxin-free plasmid constructs using Lipofectamine (Invitrogen,11514-015) according to the manufacturer's procedure.

\section{Blood RNA extraction and RT-qPCR}

$10 \mathrm{ml}$ blood from each subject was drawn with a BD Vacutainer CPT Cell Preparation Tube containing sodium citrate. Lymphocytes and monocytes were separated from the plasma in Ficoll solution (Sigma, F2637). Briefly, the blood samples were diluted with 1:1 sterile PBS, and then carefully poured onto $10 \mathrm{ml}$ Ficoll solution in a $50 \mathrm{ml}$ centrifuge tube (the blood must remain on top, do not mix). The tubes were centrifuged for $20 \mathrm{~min}$ at $350 x g$, and lymphocytes and monocytes between plasma and Ficoll layers were harvested using a sterile pipette. The cells were washed twice with PBS, and the RNA was extracted using the RNAiso reagent (Takara,9109) to template cDNA synthesis using the PrimeScript RT reagent kit (Takara,RR014). SYBR green real-time qPCR reactions (Vazyme,Q111) were carried out on a ABI7500 Real-Time PCR system. The cycling condition was $95^{\circ} \mathrm{C}$ for 5 minutes, followed by 40 amplification cycles of $95^{\circ} \mathrm{C}, 15$ second and $60^{\circ} \mathrm{C}, 1$ minute. For each data point, triplicate reactions were carried out and the experiment was repeated three times to assess the statistical significance. RT-qPCR primer sequences are listed as follows.

PCR primers for human genes

hNRAS-F: AACAAGCCCACGAACT

hNRAS-R: TGGCAATCCCATACAA

hCBL-F: CCACTTGCTCGTCTCC

hCBL-R: AACAGTAGTATCCCACATC

hBRAF-F: CCTCATTACCTGGCTCAC

$\mathrm{h} B R A F-\mathrm{R}:$ TCTCCCAATCATCACTCG

hRAF1-F: GTCACGCTGGAGTGGTTCT

hRAF-R: ACAATACGATGCCATAGGAGT

hSHOC2-F: TTTTGTCCAGGCTTGAGT

hSHOC2-R: CATCTTTGGCATCTTTCC

hSOS1-F: CTTAGGTGGAGGTGAGAA

hSOS1-R: TGGTCCCTGATTAAATAGA

hPTPN11-F: TATCCTCTGAACTGTGCAGATCC

hPTPN11-R: TCTGGCTCTCTCGTACAAGAAAA

hDNM3-F: AATCCGTCCACTAGAATCCTCA

hDNM3-R: GGTCCATACATGCGACTACTCA

hDNM3OS-F: GGTCTCACCCTGCTTGTTAATCAA

hDNM3OS-R: TCCTGTTGTTACTGGCCCTCATGC

PCR primers for mouse genes

mNras-F: CCTTGACCCGTTTGACACT

mNras-R: AACCACCTACATACCTACAT

mCbl-F: AGGGTTTCACCGTCTT 
mCbl-R: CTGGGCTGAGTGTAGTTT mBraf-F: ACCTCGTCACAGTTCTCCT mBraf-R: TTCTTGGCTTGAAGTTGC mRaf-F: TGCGTCGGATGCGAGAAT mRaf1-R: TGAGGAAGGGCTGGAGG mShoc2-F: TCGCTTTAATCGCATAAC mShoc2-R: TGAGCTACATCCAGGGTA mPtpn11-F: GAGGAGTCGATGGCAGTT mPtpn11-R: CTGAATCTTGATGTGGGTAA mMmp13-F: GTTGACAGGCTCCGAGAAAT mMmp13-R: CATCAGGCACTCCACATCTT mCol10a1-F: AAGGAGTGCCTGGACACAAT mCol10a1-R: ATGCCTGGGATCTTACAGGT mSox9-F: CGGAACAGACTCACATCTCTCC mSox9-R: GCTTGCACGTCGGTTTTGG mCol1a1-F: GCTCCTCTTAGGGGCCACT mCol1a1-R: CCACGTCTCACCATTGGGG mCol2a1-F: GGGTCACAGAGGTTACCCAG mCol2a1-R: ACCAGGGGAACCACTCTCAC mDnm3os-F: CAAGGCTCTCACTTGTCCTG mDnm3os-R: CAGCTGGAAACTGACCAAAG mLfi35-F: GTGACCCTGCAAACTGTCCTC mLfi35-R: TTCAGGTACTGAGAATGGGATCT mLfi47-F: TCTCCAGAAACCCTCACTGGT mLfi47-R: TCAGCGGATTCATCTGCTTCG mLfit1-F: CTGAGATGTCACTTCACATGGAA mLfit1-R: GTGCATCCCCAATGGGTTCT mLifh1-F: AGATCAACACCTGTGGTAACACC mLifh1-R: CTCTAGGGCCTCCACGAACA mStat1-F: TCACAGTGGTTCGAGCTTCAG mStat1-R: GCAAACGAGACATCATAGGCA mSfrp2-F: CGTGGGCTCTTCCTCTTCG mSfrp2-R: ATGTTCTGGTACTCGATGCCG mRsad2-F: GCAGAGATGGACGATATGAGAGG mRsad2-R: GCTGAGTGCTGTTCCCATCT mNmi-F: GCAGAGATGGACGATATGAGAGG mNmi-R: CGACTGCAATTCAGCTTCAAGTT mNgf-F: TGATCGGCGTACAGGCAGA mNgf-R: GCTGAAGTTTAGTCCAGTGGG mMmp3-F: ACATGGAGACTTTGTCCCTTTTG mMmp3-R: TTGGCTGAGTGGTAGAGTCCC mLif-F: GCCCCAGAAGTAAAACCTTCAG mLif-R: CCTTCCATTTCTCTCCATTCCAA mFlt4-F: CTGGCAAATGGTTACTCCATGA mFlt4-R: ACAACCCGTGTGTCTTCACTG mCxcl10-F: CCAAGTGCTGCCGTCATTTTC mCxcl10-R: GGCTCGCAGGGATGATTTCAA mMmp9-F: CTGGACAGCCAGACACTAAAG mMmp9-R: CTCGCGGCAAGTCTTCAGAG

\section{Immunofluorescence staining and RNA-FISH}

Cultured cells were fixed in 4\% paraformaldehydein PBS for $15 \mathrm{~min}$ at room temperature, and blocked for $30 \mathrm{~min}$ in PBS, 3\%BSA (Biofroxx,4240), and 0.3\% Triton X-100 (Biosharp, BS084) prior to overnight incubation with primary antibodies. Anti-Sox9 (Cell Signaling Technology, D8G8H), anti-Col2a1 (Bioss Antibodies, bs-10589R), anti-Mmp13 (Bioss Antibodies, bs-10581R) and Collagen10a1 (Biorbyt, orb221376) were used at 1:1000. Cells grown on cover slips for RNA-FISH were fixed in $3.7 \%$ formaldehyde solution at room temperature for 10 minutes, then permeabilized with $70 \%$ cold ethanol for at least an hour. Stellaris probe was added in $100 \mu \mathrm{L}$ of hybridization buffer (Biosearch, Inc.) and incubated in a dark humidified chamber at $37^{\circ} \mathrm{C}$ for $4 \mathrm{~h}$. The cells were visualized with DAPI (Sigma,MBD0015) counterstaining $(5 \mathrm{ng} / \mathrm{mL})$ under a wide field fluorescence microscope.

\section{Edu incorporation assay}

To measure cell growth, 24 hours after transfection in 24-well plates, $20 \mathrm{mM}$ EdU (Ribobio,C10310-1) was added for 8 hour. The cells were then fixed in $3.7 \%$ formaldehyde, then washed with PBS and permeablized. $500 \mu \mathrm{l}$ Click-iT reaction cocktail $(430 \mathrm{ml}$ 1xClickiT reaction buffer, $20 \mathrm{ml} \mathrm{CuSO}_{4}, 1.2 \mathrm{ml}$ Alexa Fluor ${ }^{\circledR}$ azide, and $50 \mathrm{ml}$ reaction buffer additive) was added to each well and incubated for $45 \mathrm{~min}$ at the room temperature in the dark. The cells were counter-stained with DAPI for nuclei and visualized under an inverted fluorescence microscope. Images were processed with Image $J$ and the percentage of EdU incorporation was calculated based on the number of EdU positive (red) and total (DAPI) cells.

\section{Alcian blue staining and quantification}

Chondrocytes were cultured for 14 days in chondrogenic differentiation medium, then fixed in $4 \%$ formalin for 10 min. After washing twice with PBS, the cells were incubated with $3 \%$ acetic acid for $10 \mathrm{~min}$ and stained with $1 \%$ alcian blue in $3 \%$ acetic acid ( $\mathrm{pH} \mathrm{2.5)}$ for $30 \mathrm{~min}$ and photographed. For quantification, the stained cells were washed twice, and the alcian blue dye was extracted with $500 \mathrm{ml}$ dimethyl sulfoxide (Sigma,D8418). Absorbance was measured at $650 \mathrm{~nm}$.

\section{Alkaline phosphatase assay}

Histochemical detection of alkaline phosphatase activity was performed on cells that were fixed for $2 \mathrm{~min}$ in $4 \%$ paraformaldehyde at room temperature. After washing with TBST, the cells were incubated for 30 min in in $0.1 \mathrm{M}$ Tris- $\mathrm{HCl}, \mathrm{pH} 8.5$, containing $0.1 \mathrm{mg} /$ $\mathrm{ml}$ Naphthol AS-MX phosphate (Sigma,N4875), 0.5\% $\mathrm{N}, \mathrm{N}$-dimethylformamide (Sigma,D4551), $2 \mathrm{mM} \mathrm{MgCl}{ }_{2}$, and $0.6 \mathrm{mg} / \mathrm{ml}$ fast blue BB salt (Sigma,D9805), and then photographed. 


\section{In vitro differentiation of chondrogenic ATDC5 cells} ATDC5 cell line was culture in DMEM/F-12 medium supplemented with 5\% FBS, penicillin (100units $/ \mathrm{mL}$ )/ streptomycin $(0.1 \mathrm{mg} / \mathrm{mL})$, and $4 \mathrm{mM} \mathrm{L-Glutamine}$ (Gibco,25030-081). For differentiation experiments, ATDC5 cells were seeded at $80-90 \%$ confluence in 12 -well plate. After reaching 100\% confluence, ATDC5 cells were incubated with serum-free medium for $24 \mathrm{~h}$, then exposed to differentiating medium containing $1 \%$ Insulin-transferrin-sodium selenite (ITS, Sigma-Aldrich) and 50nM Vitamin C (Sigma,A4544).

\section{RNA sequencing and data analysis}

RNA from Rk5-vector, Full length and DKO mutant Dnm3os transfected primary mouse articular chondrocytes were extracted using TRIzol (Life Technol- ogies), followed by purification using a RNeasy Mini Kit (Qiagen,74104). RNA-seq was performed using primary mouse articular chondrocytes from two individual animals. RNA-seq libraries were prepared using the Illumina TruSeq RNA Library Prep Kit v2 and sequenced by a HiSeq 2500 sequencer. RNA-seq reads were aligned to mm10 using TopHat with default settings (.cbcb.umd. $\mathrm{edu} /)$.

\section{Statistical analysis}

Prism 8.0 (GraphPad software) was employed for analyses. Data from a minimum of three independent experiments were presented as mean \pm s.d. Animals in the experiment were randomly selected and grouped. An unpaired two-tailed Student's t-test was used for analyzing two data sets, and one-way analysis of variance was used for more sets. The significance threshold was set at $0.05(p<0.05)$.

\section{Supplementary Information}

The online version contains supplementary material available at https://doi. org/10.1186/s13578-021-00559-8.

\footnotetext{
Additional file 1. Clinical description of the proband.

Additional file 2: Fig. S1. Body weight, body length and morphometric characteristics skull in miR-214 KO mice. Body weight (A) and body length (B) of miR-214 KO mice $(n=15)$ and their WT littermates $(n=18)$ measured starting from 6 week to 24 weeks of age. Body weight and body length of the KO mice were modestly smaller than the WT mice at most time-points examined, the difference was not statistically significant. Morphometric characteristics skull width(C), skull length (D), skull width/ length, and inner canthal distance (E) of WT and miR-214 KO, the difference was not statistically significant.
}

Additional file 3: Fig. S2. miR-214 KO drastically increase the height relative genes. QPCR expression analysis of Noonan syndrome relative genes in miR-214 KO blood, miR-214 Het relative to WT mice ( $n=5$ in each genotype). Data shown are the fold induction of gene expression normalized with Hprt and expressed as mean \pm S.E.M. One-way ANOVA test was used for statistical analysis. ${ }^{* *} \mathrm{P}<0.01,{ }^{* * *} \mathrm{P}<0.001$, and $\mathrm{ns}$, not significant
Additional file 4: Table S1. Predicted miR-214 and miR-199a recognition sites.

Additional file 5: Fig. S3. LncRNA-Dnm3os impedes chondrocyte differentiation while promotes the proliferation. RT-qPCR quantification of Sox9 in primary articular chondrocytes at passages as noted. (B) RT-qPCR quantification and statistical analysis of Col1a1 and Col2a1 in primary articular chondrocytes transfected with shDnm3os at passages as noted. (C) EdU labeling of proliferating chondrocytes transfected with scrambled shRNA, shDnm3os and shDnm3os together with DKO. (D) RT-qPCR quantification of Dnm3os in (C). (E) statistical analysis of EdU positive cells in (C). (F) RT-qPCR quantification of Col10a1 and (G) Mmp13 in primary articular chondrocytes transfected with scrambled shRNA, shDnm3os and shDnm3os together with DKO after 2 weeks chondrocyte differentiation. $(H-l)$ RT-qPCR quantification of overexpression level of Dnm3os in primary articular chondrocytes and ATDC5 cells transfected with RK5, FL and DKO. ${ }^{*}, P<0.05,{ }^{* *}, P<0.01, * * *, P<0.001$

Additional file 6: Fig. S4. Forced expression of miR-214 and miR-199a impedes chondrocyte differentiation while promotes the proliferation. Confocal images of GFP and IF staining of Col2al in primary articular chondrocytes after the cells were transfected with P2GM vector, P2GMmiR-1 99a (P199), or P2GM-miR214 (P214) and differentiated for 2 weeks. (B) RT-qPCR quantification of Col2a1 or (C) Col10a1 in cells of (A). (D) the same as in (A) except for the staining of Sox9 and the cells were quantified for Sox9 (E) and (F) Mmp13 by RT-qPCR. (G) and (H) EdU staining of cells as in (A) and quantification thereof. Student T-test was used for statistical analysis. ${ }^{*}, P<0.05,{ }^{* *}, P<0.01,{ }^{* * *}, P<0.001$.

Additional file 7: Fig. S5. Forced expression of Dnm3os or Dnm3os-DKO downregulates height-related genes in mouse primary articular chondrocytes. RT-qPCR analysis of Noonan syndrome genes in primary chondrocytes that were transfected with pRK5-Dnm3os or pRK5-Dnm30sDKO. Data shown are the fold induction normalized against $\mathrm{Hprt}$ and are expressed as mean \pm S.E.M. One-way ANOVA test was used for the statistical analysis. ${ }^{* *} \mathrm{P}<0.01$, ${ }^{* *} \mathrm{P}<0.001$, and ns, not significant.

Additional file 8: Fig. S6. Insulin-supplemented differentiation medium cultivation induces differentiation of ATDC5 cells. (A) Alcian blue staining of cartilage matrix produced by differentiated ATDC 5 cells after 18-days differentiation. (B-C) RT-qPCR quantifications of Col1a1, Col2a1 in ATDC5 cells at different days of differentiation as noted. (D) RT-qPCR quantifications of Dnm3os in ATDC5 cells at different days of differentiation as noted. (E) RT-qPCR quantifications of Sox9 in ATDC5 and iMAC cells transfected with RK5, FL and DKO. (G) Top 20 enriched pathways in FL and DKO transfected primary articular chondrocytes. The size and color of the dots represent the enriched gene number and the range of $-\log 10$ ( $p$ values), respectively. Student T-test was used for statistical analysis. ${ }^{*}, P<0.05,{ }^{* *}$, $P<0.01, * * *, P<0.001$.

Additional file 9: Table S2. shRNA sequence of Dnm3os.

\section{Abbreviations}

MAPK: Mitogen-activated protein kinase; ncRNA: Noncoding RNA; IncRNAs: Long non-coding RNAs; NGF: Nerve growth factor; DNM3: Dynamin 3; Dnm3os: DNM3 opposite strand; TMJ: Temporomandibular joint; MSCs: Mesenchymal stem cells.

\section{Acknowledgements}

We wish to thank the family for participating in this study and other members of the Research Team at Children's Hospitals and Clinics of Minnesota.

\section{Authors' contributions}

SY, CL and SYC conceived of the presented idea. TY, QX, SL, HH and LS performed the research. $\mathrm{XL}$ and $\mathrm{HL}$ contributed to sample preparation and result analysis. SD collected the clinical case and tested the clinical sample. JAR and $\mathrm{BAH}$ contributed to the genetic testing and interpreted the results. All authors read and approved the final manuscript. 


\section{Funding}

This work was supported by grants from the Chinese National Science foundation $(81272238,81261120386,81672748$ and 81871936$)$ to SYC. TTY is supported by a young investigator grant from the Chinese National Science Foundation (81602431). SY is supported by a grant from the Chinese National Science Foundation (81572720). CL is supported by Natural Science Foundation of Jiangsu Province (BK20171053) and National Natural Science Funds of China (81702747).

\section{Availability of data and materials}

All data generated or analysed during this study are included in this published article and its additional files.

\section{Ethics approval and consent to participate}

The animal study was reviewed and approved by the Institutional Animal Care and Use Committee of Nanjing Medical University.

\section{Consent for publication}

Not applicable.

\section{Competing interests}

The authors declare that they have no competing interests.

\section{Author details}

${ }^{1}$ Department of Medical Genetics, School of Basic Medical Sciences, Nanjing Medical University, Jiangsu 211166 Nanjing, P. R. China. ${ }^{2}$ Department of Medical Genetics, Children's Hospital and Clinics of Minnesota, Minneapolis, Ml 55404, USA. ${ }^{3}$ Department of Neurology, Ohio State University Medical Center, Columbus, OH 43210, USA. ${ }^{4}$ University of Minnesota Medical Center-Fairview, Minneapolis, MI 55404, USA.

Received: 11 October 2020 Accepted: 17 February 2021

Published online: 02 March 2021

\section{References}

1. Nilsson O, Marino R, De Luca F, et al. Endocrine regulation of the growth plate. Horm Res. 2005;64(4):157-65.

2. Xing W, Godwin C, Pourteymoor S, et al. Conditional disruption of the osterix gene in chondrocytes during early postnatal growth impairs secondary ossification in the mouse tibial epiphysis. Bone Res. 2019;7:24.

3. Wit JM, Deeb A, Bin-Abbas B, et al. Achieving optimal short- and longterm responses to paediatric growth hormone therapy. J Clin Res Pediatr Endocrinol. 2019;11(4):329-40.

4. Jee YH, Baron J. The biology of stature. J Pediatr. 2016:173:32-8.

5. Baron J, Savendahl L, De Luca F, et al. Short and tall stature: a new paradigm emerges. Nat Rev Endocrinol. 2015;11(12):735-46.

6. Misra M, Klibanski A. Endocrine consequences of anorexia nervosa. Lancet Diabetes Endocrinol. 2014;2(7):581-92.

7. Yao B, Wang Q, Liu CF, et al. The SOX9 upstream region prone to chromosomal aberrations causing campomelic dysplasia contains multiple cartilage enhancers. Nucleic Acids Res. 2015:43(11):5394-408.

8. Stanton LA, Underhill TM. Beier F MAP kinases in chondrocyte differentiation. Dev Biol. 2003;263(2):165-75.

9. Jost M, Huggett TM, Kari C, et al. Epidermal growth factor receptordependent control of keratinocyte survival and BCl-xL expression through a MEK-dependent pathway. J Biol Chem. 2001;276(9):6320-6.
10. Furstenberger $\mathrm{G}$, Senn HJ. Insulin-like growth factors and cancer. Lancet Oncol. 2002;3(5):298-302.

11. Ornitz DM, Itoh N. The Fibroblast Growth Factor signaling pathway. Wiley Interdiscip Rev Dev Biol. 2015;4(3):215-66.

12. Tidyman WE, Rauen KA. The RASopathies: developmental syndromes of Ras/MAPK pathway dysregulation. Curr Opin Genet Dev. 2009:19(3):230-6.

13. Ling $H$, Fabbri M, Calin GA. MicroRNAs and other non-coding RNAs as targets for anticancer drug development. Nat Rev Drug Discov. 2013;12(11):847-65.

14. Burkardt DD, Rosenfeld JA, Helgeson ML, et al. Distinctive phenotype in 9 patients with deletion of chromosome 1q24-q25. Am J Med Genet A. 2011;155A(6):1336-51.

15. Descartes M, Hain JZ, Conklin M, et al. Molecular characterization of a patient with an interstitial 1q deletion [del(1)(q24.1q25.3)] and distinctive skeletal abnormalities. Am J Med Genet A. 2008;146A(22):2937-43.

16. Watanabe T, Sato T, Amano T, et al. Dnm3os, a non-coding RNA, is required for normal growth and skeletal development in mice. Dev Dyn. 2008;237(12):3738-48.

17. Liu J, Luo XJ, Xiong AW, et al. MicroRNA-214 promotes myogenic differentiation by facilitating exit from mitosis via down-regulation of protooncogene N-ras. J Biol Chem. 2010;285(34):26599-607.

18. Aurora AB, Mahmoud Al, Luo X, et al. MicroRNA-214 protects the mouse heart from ischemic injury by controlling $\mathrm{Ca}(2)(+)$ overload and cell death. J Clin Invest. 2012;122(4):1222-32.

19. Huang HJ, Liu J, Hua H, et al. MiR-214 and N-ras regulatory loop suppresses rhabdomyosarcoma cell growth and xenograft tumorigenesis. Oncotarget. 2014;5(8):2161-75.

20. Loebel DA, Tsoi B, Wong $\mathrm{N}$, et al. A conserved noncoding intronic transcript at the mouse Dnm3 locus. Genomics. 2005;85(6):782-9.

21. Long F, Ornitz DM. Development of the endochondral skeleton. Cold Spring Harb Perspect Biol. 2013;5(1):a008334.

22. Gosset M, Berenbaum F, Thirion S, et al. Primary culture and phenotyping of murine chondrocytes. Nat Protoc. 2008;3(8):1253-60.

23. Lu Z, Lei $D$, Jiang $T$, et al. Nerve growth factor from Chinese cobra venom stimulates chondrogenic differentiation of mesenchymal stem cells. Cell death disease. 2017;8(5):e2801.

24. Li ZZ, Wang F, Liu S, et al. Ablation of PKM2 ameliorated ER stress-induced apoptosis and associated inflammation response in IL-1beta-treated chondrocytes via blocking Rspo2-mediated Wnt/beta-catenin signaling. J Cell Biochem. 2020;5:25.

25. Bach FC, de Rooij KM, Riemers FM, et al. Hedgehog proteins and parathyroid hormone-related protein are involved in intervertebral disc maturation, degeneration, and calcification. JOR Spine. 2019;2(4):e1071.

26. Ashraf T, Collinson MN, Fairhurst J, et al. Two further patients with the 1q24 deletion syndrome expand the phenotype: a possible role for the miR199-214 cluster in the skeletal features of the condition. Am J Med Genet A. 2015;167A(12):3153-60.

27. Desvignes T, Contreras A, Postlethwait JH. Evolution of the miR199-214 cluster and vertebrate skeletal development. RNA Biol. 2014;11(4):281-94

\section{Publisher's note}

Springer Nature remains neutral with regard to jurisdictional claims in published maps and institutional affiliations.

Ready to submit your research? Choose BMC and benefit from:

- fast, convenient online submission

- thorough peer review by experienced researchers in your field

- rapid publication on acceptance

- support for research data, including large and complex data types

- gold Open Access which fosters wider collaboration and increased citations

- maximum visibility for your research: over 100M website views per year

At $\mathrm{BMC}$, research is always in progress.

Learn more biomedcentral.com/submissions 